\title{
Avaliação do grau de dependência tabágica e o grau de adequação das orientações recebidas por pacientes tabagistas hospitalizados
}

\author{
Evaluation of the degree of tobacco addiction and the adequacy of orientation \\ received by hospitalized smokers
}

Gabriel Bordignon ${ }^{1}$, Roddie Moraes Neto ${ }^{1}$, Gustavo Lenci Marques ${ }^{2}$

Bordignon G, Moraes Neto R, Marques GL. Avaliação do grau de dependência tabágica e o grau de adequação das orientações recebidas por pacientes tabagistas hospitalizados / Evaluation of the degree of tobacco addiction and the adequacy of orientation received by hospitalized smokers. Rev Med (São Paulo). 2020 maio-jun.;99(3):251-7.

RESUMO: Introdução: o tabagismo é uma doença crônica e um grande fator de risco para outras doenças com alta mortalidade. É dever do médico orientar corretamente seus pacientes quanto à cessação do tabagismo. Para tanto, as orientações devem ser embasadas cientificamente para manejo eficaz da doença, com intervenções motivacionais associadas ou não a farmacoterapia. Objetivo: analisar o grau de dependência da nicotina de pacientes tabagistas hospitalizados e comparar as orientações recebidas sobre cessação de tabagismo com a orientação farmacológica e não farmacológica preconizada pela literatura. Métodos: estudo observacional transversal com aplicação de questionário estruturado a 60 pacientes tabagistas no momento da alta hospitalar do Complexo Hospital de Clínicas da Universidade Federal do Paraná (CHC/UFPR). Resultados: amostra de pacientes composta por 31 pacientes do sexo feminino e 29 do sexo masculino. $68,3 \%$ possuíam mais de 55 anos. A mediana do grau de dependência nicotínica foi 5 (grau médio). Houve diferença estatisticamente significativa entre grupos com orientação presente e ausente em relação ao grau de motivação. $44,2 \%$ dos pacientes que receberam orientação estavam em fase de ação, indicando que o médico orienta mais os que já iniciaram planejamento de cessar o tabagismo. 35,3\% dos pacientes com orientação ausente estavam em pré-contemplação e 41,2\% em contemplação, sugerindo que pacientes que não possuem insight de cessar o tabagismo são menos orientados. Entre os grupos orientados de forma correta e incorreta, houve diferença estatisticamente significativa quanto ao tipo de orientação durante a hospitalização. Dentre os orientados de forma incorreta, 18 foram orientados apenas de forma motivacional, indicando uma tendência de orientação insuficiente. Conclusão: 51,2\% das orientações são realizadas incorretamente, expressando necessidade de reavaliar a abordagem e tratamento do tabagismo. Faz-se necessária busca ativa dos tabagistas durante atendimento médico e orientação correta para tratamento eficiente da doença, abordando o lado motivacional e a farmacoterapia.

Descritores: Abandono do uso de tabaco; Tabagismo; Hospitalização.
ABSTRACT: Introduction: Smoking is a chronic disease and a major risk factor for other diseases with high mortality. It's physician's duty to properly guide their patients to quit smoking. For that, the orientations must be scientifically based for effective management of the disease, with motivational interventions with or without pharmacotherapy. Objective: analyze the dependence degree of hospitalized smokers, using Fagerström test, comparing received orientations about quit smoking with pharmacological and non-pharmacological orientation present in literature. Methods: cross-sectional observational study with structured questionnaire applied to 60 smokers at the moment of hospital discharge from "Complexo Hospital de Clínicas da Universidade Federal do Paraná" (CHC/UFPR). Results: The patient sample was composed of 31 male patients and 29 female patients. $68.3 \%$ of the patients had more than 55 years. The median of the dependence degree was 5 (average degree). There was statistically significant difference between groups with present and absent orientation, regarding to the degree of motivation. $44.2 \%$ of the patients who had received orientation were in action phase, indicating that physicians guide more patients who already begun planning quit smoking. $35.3 \%$ of the patients with absent orientation were in pre-contemplation and $41.2 \%$ were in contemplation, suggesting that patients who don't have an insight to quit smoking are less oriented. Between correctly and incorrectly oriented groups, there was statistically significant difference in the type of hospital guidance. Among incorrectly oriented, 18 were oriented only motivationally, indicating an insufficient orientation trend. Conclusions: $51,2 \%$ of the orientations are performed incorrectly, expressing the need to re-evaluate the approach and treatment of smoking. There is the need of active search of smokers during the medical care and correct orientation for efficient treatment of the disease, addressing motivational side and pharmacotherapy.

Keywords: Tobacco use cessation; Tobacco use disorder; Hospitalization.

Trabalho realizado no Complexo Hospital de Clínicas da Universidade Federal do Paraná (CHC-UFPR).

1. Acadêmicos do curso de Medicina da Universidade Federal do Paraná, Curitiba-PR. ORCID: Gabriel Bordignon - https://orcid.org/0000-0002-98344762; Roddie Moraes Neto - https://orcid.org/0000-0002-4936-592X. Email: gbordela@gmail.com; roddie.moraes@gmail.com.

2. Mestre em medicina interna. Professor adjunto do Departamento de Medicina Interna da Universidade Federal do Paraná, Curitiba, PR. ORCID: https://orcid.org/0000-0002-6057-0350. Email: gustavolencimarques@gmail.com.

Endereço para correspondência: Roddie Moraes Neto. Rua Jovino do Rosário, nº 475, Boa Vista. Curitiba, PR, BR. CEP: 82540-115. Email: roddie. moraes@gmail.com 


\section{INTRODUÇÃO}

$\mathrm{O}$ tabagismo, além de ser uma doença crônica devido a dependência da nicotina, é um dos maiores fatores de risco para outras doenças e está relacionado à alta mortalidade ${ }^{1}$. O tabagismo responde por $45 \%$ das mortes por infarto, $85 \%$ das mortes por doença pulmonar obstrutiva crônica (DPOC), 25\% das mortes por doenças cerebrovasculares (AVE) e 30\% das mortes por câncer. Em relação ao último, destaca-se que $90 \%$ dos casos de câncer de pulmão ocorrem em tabagistas ${ }^{2}$. Ao todo, estão estabelecidas formalmente 21 doenças causadas pelo tabagismo (12 tipos de câncer, 6 categorias de doença cardiovascular, diabetes, DPOC e pneumonia, incluindo influenza) ${ }^{3}$.

Dados da Pesquisa Nacional de Saúde de 2013 indicaram a prevalência de aproximadamente 21 milhões de pessoas fumantes de cigarro. Dentre esses fumantes, $51 \%$ haviam tentado parar de fumar nos 12 últimos meses anteriores à pesquisa, porém somente $5 \%$ havia procurado tratamento, o que mostra uma baixa procura especializada para o tratamento da dependência do tabagismo $0^{4,5}$.

Dados obtidos pela Vigilância de Fatores de Risco e Proteção para Doenças Crônicas por Inquérito Telefônico (Vigitel) de 2018 indicaram uma frequência de fumantes de $9,3 \%$, sendo quase duas vezes mais prevalente no sexo masculino do que no sexo feminino. A cidade onde o presente estudo foi realizado, Curitiba, estava em $3^{\circ}$ lugar dentre as maiores frequências de fumantes dentre as capitais dos 26 estados brasileiros e o Distrito Federal ${ }^{6}$.

Além de todo impacto social e biológico causado pelo tabagismo, o custo para o sistema de saúde é um importante ponto, o que evidencia a necessidade de correta orientação médica e multidisciplinar afim de obter um tratamento com alta efetividade, visando diminuir morbidade, mortalidade e custos gerados pelo tabagismo. Em um estudo que analisou o custo do tabagismo para o sistema de saúde, estimou-se que cerca de $8 \%$ de todo o gasto em saúde (aproximadamente 39 bilhões de reais) foi destinado à assistência médica atribuível ao tabagismo, dando destaque à DPOC, doenças cardíacas e neoplasias - principalmente câncer de pulmão -, como doenças que apresentaram os maiores custos diretos ${ }^{7}$.

Dado o conhecimento da dependência do tabagismo como doença, é preciso encarar o tratamento como uma forma de cessar o tabagismo e prevenir possíveis recaídas, tanto com intervenções motivacionais, principalmente nos métodos baseados na terapia comportamental cognitiva (TCC), quanto com a farmacoterapia. Este último é um recurso adicional que visa diminuir a síndrome de abstinência - caracterizada por sinais e sintomas desagradáveis que surgem ao parar de fumar, os quais variam com o grau de dependência da nicotina - para aumentar o sucesso terapêutico ${ }^{8,9}$. Os medicamentos utilizados como primeira linha no tratamento da dependência da nicotina, com alta segurança, são a Terapia de Reposição da Nicotina (TRN), Vareniclina e a Bupropiona ${ }^{10}$.

Inúmeros são os estudos que demonstraram a eficácia da TRN como droga adjuvante para cessação do tabagismo $^{10}$. Metanálises mostraram que a TRN quase dobrou o risco relativo do paciente alcançar a cessação do hábito quando comparado ao placebo, concluindo que esse tratamento aumenta a chance de o paciente parar de fumar ${ }^{11,12}$.

Para a decisão do planejamento terapêutico na cessação do tabagismo é imprescindível a avaliação do grau de dependência da nicotina, o qual pode ser realizado por diferentes métodos. Dentre eles, destaca-se o Teste de Fagerström para a dependência da nicotina, que consiste em uma ferramenta composta de seis perguntas, havendo uma pontuação para cada resposta. A somatória das pontuações pode classificar os pacientes em muito baixo, baixo, médio, elevado e muito elevado grau de dependência à nicotina. De acordo com essa classificação, está indicado o tratamento motivacional com farmacoterapia para pacientes com pontuações igual ou superior a cinco (grau médio, de acordo com o teste) ${ }^{8-10,13}$. Os pacientes tabagistas podem ser também classificados de acordo com o grau de motivação quanto à intenção da cessação do tabagismo. Para tanto, utiliza-se a classificação nos estágios de pré-contemplação, contemplação, preparação, ação e manutenção. Esses estágios correspondem, respectivamente, ao tabagista que ainda não manifesta a intenção de parar de fumar, ao que manifesta essa intenção, ao tabagista que manifesta intenção e já possui planejamento concreto para tal, ao que já cessou o tabagismo e, por fim, ao que parou de fumar a pelo menos seis meses ${ }^{14}$.

Dada as morbidades relacionadas ao tabagismo, frequentemente os pacientes tabagistas ativos são hospitalizados por alguma doença relacionada ao tabaco. Os fumantes hospitalizados geralmente são mais suscetíveis às orientações contra o tabaco. Sabe-se que a combinação da intervenção motivacional aliada à farmacoterapia com TRN, após a alta hospitalar, aumenta a taxa de cessação se comparada à intervenção motivacional isolada durante a hospitalização. Portanto, faz-se necessária uma abordagem intensificada nesse perfil de paciente, dado que há uma maior disposição para cessarem o tabagismo ${ }^{13,15-17}$.

\section{OBJETIVO}

Este estudo teve como objetivo analisar o grau de dependência da nicotina dos pacientes tabagistas hospitalizados no Complexo Hospital de Clínicas da Universidade Federal do Paraná - Curitiba - PR e comparar as orientações recebidas por esses pacientes durante a hospitalização sobre cessação de tabagismo com a orientação farmacológica e não farmacológica preconizada pela literatura.

\section{MÉTODO}


Para realizar a análise proposta foi conduzido um estudo observacional transversal descritivo no Complexo Hospital de Clínicas da Universidade Federal do Paraná (CHC/UFPR), localizado na cidade de Curitiba, capital do estado do Paraná. A amostragem do estudo foi composta de 60 pacientes tabagistas hospitalizados no CHC/UFPR, sendo que os questionários foram aplicados no dia em que estes pacientes receberam alta hospitalar, no período de janeiro a agosto de 2019.

O estudo foi iniciado apenas após a aprovação do projeto de pesquisa pelo Comitê de Ética em Pesquisa em Seres Humanos do Complexo Hospital de Clínicas da Universidade Federal do Paraná, sob protocolo CAEE 99748018.0.0000.0096, número do parecer 3.737.758. Todos os participantes assinaram o Termo de Consentimento Livre e Esclarecido (TCLE).

$\mathrm{O}$ instrumento utilizado no estudo foi um questionário elaborado pelos pesquisadores contendo 11 perguntas, como: sexo, idade, grau de instrução, comorbidades, motivo de hospitalização, presença ou não na UTI, grau de motivação para cessação do tabagismo, carga tabágica em anos-maço, o que o paciente sabia sobre cessação do tabagismo, orientações prévias recebidas para cessação do tabagismo, e por último, orientação para cessação do tabagismo na atual hospitalização e como foi essa orientação. Além disso, foi aplicado o teste de Fagerström para avaliação do grau de dependência da nicotina. As questões foram de caráter objetivo questionando aspectos sociais, educacionais e relacionados ao hábito tabágico. A aplicação do questionário foi realizada pelos pesquisadores, em um encontro único com o paciente à beira do leito, na forma de entrevista estruturada, com duração aproximada de 30 minutos.

Com os dados obtidos, foi possível dividir a amostra em dois grupos: os que receberam e os que não receberam orientação sobre cessação do tabagismo durante a hospitalização no CHC/UFPR. Caso recebida, foi questionado se a orientação foi somente uma intervenção motivacional ou se foi realizada uma intervenção motivacional associada a farmacoterapia. Por último, esse grupo que recebeu orientação durante a hospitalização foi dividido em outros dois grupos: orientação correta e orientação incorreta, segundo a literatura. Ao final da aplicação, os pacientes foram orientados a parar de fumar, conforme a Diretriz para Cessação do Tabagismo, utilizada como base para este estudo.

Foi construído um banco de dados no Microsoft Office Excel ${ }^{\circledR}$ e os dados foram analisados. Todas as variáveis coletadas foram descritas utilizando medidas resumo. As variáveis qualitativas foram descritas através das proporções e as variáveis quantitativas através da mediana e intervalo interquartílico (primeiro quartil; terceiro quartil). Variáveis de interesse foram comparadas entre os pacientes que receberam e os que não receberam orientação prévia. A diferença entre os dois grupos de pacientes foi testada utilizando o teste de Mann-Whitney-Wilcoxon para variáveis quantitativas e usando o teste exato de Fisher para as variáveis qualitativas.
As análises foram conduzidas utilizando o software $\mathrm{R}$ de computação estatística, considerando sempre o nível de significância de 5\%. As variáveis quantitativas foram avaliadas quanto a sua normalidade pelo teste de Shapiro-Wilk e a hipótese de distribuição normal foi rejeitada em todas as ocasiões.

\section{RESULTADOS}

Foram avaliados 60 questionários de pacientes hospitalizados no CHC/UFPR no período de janeiro a agosto de 2019. Quanto a caracterização da amostra, a variável correspondente ao sexo feminino teve 31 pacientes $(51,7 \%)$, enquanto a variável do sexo masculino teve 29 pacientes (48,3\%). Observa-se que 68,3\% da amostra possuem idade acima de 55 anos. Quanto ao grau de escolaridade, 39 pacientes tinham até 8 anos de escolaridade e 21 possuíam mais que 8 anos de escolaridade.

Com relação às comorbidades, 36 pacientes $(60 \%)$ com hipertensão, 19 pacientes $(31,7 \%)$ com diabetes, 10 pacientes $(16,7 \%)$ com DPOC, 9 pacientes $(15 \%)$ com doença cerebrovascular e 11 pacientes com infarto prévio $(18,3 \%)$

No grau de motivação, 13 pacientes $(21,7 \%)$ encontraram-se em pré-contemplação, 19 (31,7\%) em contemplação, 6 (10\%) em preparação, 21 (35\%) em ação e $1(1,7 \%)$ em manutenção. O cálculo do número de anos-maço foi realizado para os 60 pacientes incluídos neste estudo com uma mediana de 36.

Na tabela de análise descritiva do perfil amostral (Tabela 1), pode-se observar as variáveis em questão, o número de pacientes correspondente a cada variável, assim como a proporção/mediana nos quartis um e três.

Dos 60 pacientes entrevistados, 44 deles $(73,3 \%)$ relataram ter conhecimento sobre o tratamento farmacológico para cessação do tabagismo, enquanto 56 pacientes $(93,3 \%)$ possuíam conhecimento sobre os riscos do tabagismo.

Um total de 48 pacientes $(80 \%)$ recebeu orientação no período pré-hospitalização, sendo que 31 (64,6\%) receberam orientação somente motivacional e $17(35,4 \%)$ receberam orientação motivacional associada à farmacoterapia. Do mesmo modo, 43 pacientes $(71,7 \%)$ receberam orientação durante a hospitalização no CHC/UFPR, sendo que $28(65,1 \%)$ receberam orientação motivacional e 15 (34,9\%) receberam orientação motivacional associada à farmacoterapia. Dos 43 pacientes que receberam orientação durante a hospitalização, 21 (48,8\%) foram orientados de maneira correta.

Na Tabela 2 encontram-se os resultados referentes à orientação prévia e à orientação durante a hospitalização recebida pelos pacientes.

Na Tabela 3 encontram-se os resultados do teste de Fagerström e os resultados para cada pergunta. A soma da pontuação do teste de Fagerström foi realizada a todos os pacientes participantes do estudo com a mediana de 5, o que indica uma dependência de nicotina em grau médio na população estudada. 
Tabela 1 - Descrição do perfil amostral ( $n=60)$

\begin{tabular}{l|l|c|c}
\hline \multicolumn{2}{l|}{ Variável } & Número de pacientes & Proporção/mediana (Q1;Q3) \\
\hline \multirow{2}{*}{ Sexo } & Feminino & 31 & $51,7 \%$ \\
& Masculino & 29 & $48,3 \%$ \\
\hline \multirow{4}{*}{ Faixa etária (anos) } & $18-35$ & 2 & $3,3 \%$ \\
& $36-54$ & 17 & $28,3 \%$ \\
& $55-64$ & 21 & $35,0 \%$ \\
\hline \multirow{2}{*}{ Grau de escolaridade } & Acima de 65 & 20 & $33,3 \%$ \\
\hline \multirow{5}{*}{ Comorbidades } & Até 8 anos de estudo & 39 & $65,0 \%$ \\
\cline { 2 - 3 } & Mais de 8 anos de estudo & 21 & $35,0 \%$ \\
& Hipertensão arterial & 36 & $60,0 \%$ \\
& Diabetes & 19 & $31,7 \%$ \\
& DPOC & 10 & $16,7 \%$ \\
& Doença cerebrovascular & 9 & $15,0 \%$ \\
& Infarto prévio & 11 & $18,3 \%$ \\
\hline \multirow{2}{*}{ Grau de motivação } & Pré-contemplação & 13 & $21,7 \%$ \\
& Contemplação & 19 & $31,7 \%$ \\
& Preparação & 6 & $10,0 \%$ \\
\hline Anos-maço & Ação & 21 & $35,0 \%$ \\
\hline
\end{tabular}

FONTE: O autor (2019).

Tabela 2 - Orientação pré-hospitalização e orientação durante a hospitalização

\begin{tabular}{|c|c|c|c|}
\hline \multicolumn{2}{|l|}{ Variável } & Número de pacientes & Proporção/mediana $(\mathrm{Q} 1 ; \mathrm{Q3})$ \\
\hline \multicolumn{2}{|l|}{ Recebeu orientação no período pré-hospitalização } & 48 & $80,0 \%$ \\
\hline \multirow{2}{*}{ Tipo de orientação recebida pré-hospitalização } & Motivacional & 31 & $64,6 \%$ \\
\hline & Motivacional + farmacoterapia & 17 & $35,4 \%$ \\
\hline \multicolumn{2}{|l|}{ Recebeu orientação durante a hospitalização } & 43 & $71,7 \%$ \\
\hline \multicolumn{2}{|l|}{ Ocorrência de orientação correta } & 21 & $48,8 \%$ \\
\hline \multirow{2}{*}{ Tipo de orientação recebida durante a hospitalização } & Motivacional & 28 & $65,1 \%$ \\
\hline & Motivacional + farmacoterapia & 15 & $34,9 \%$ \\
\hline
\end{tabular}

FONTE: O autor (2019).

Tabela 3 - Teste de Fagerström $(n=60)$

\begin{tabular}{|c|c|c|c|}
\hline \multicolumn{2}{|l|}{ Variável } & \multirow{5}{*}{ 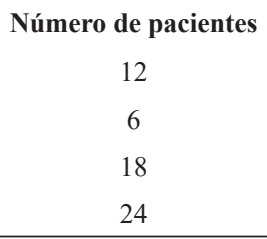 } & \multirow{5}{*}{$\begin{array}{c}\text { Proporção/mediana }(\mathbf{Q 1 ; Q 3 )} \\
20,0 \% \\
10,0 \% \\
30,0 \% \\
40,0 \%\end{array}$} \\
\hline \multirow{4}{*}{ Tempo até o primeiro cigarro } & Mais de 60 minutos & & \\
\hline & Entre 31 e 60 minutos & & \\
\hline & Entre 6 e 30 minutos & & \\
\hline & Menos de 6 minutos & & \\
\hline \multicolumn{2}{|c|}{ Dificuldade de não fumar em locais proibidos } & 36 & $60,0 \%$ \\
\hline \multicolumn{2}{|c|}{ O primeiro cigarro da manhã é o que traz mais satisfação } & 19 & $31,7 \%$ \\
\hline \multicolumn{2}{|c|}{ Fuma mais nas primeiras horas da manhã } & 10 & $16,7 \%$ \\
\hline \multicolumn{2}{|c|}{ Fuma mesmo quando acamado por doença } & 49 & $81,7 \%$ \\
\hline \multirow{4}{*}{ Cigarros por dia } & Menos de 11 & 17 & $28,3 \%$ \\
\hline & Entre 11 e 20 & 30 & $50,0 \%$ \\
\hline & Entre 21 e 30 & 5 & $8,3 \%$ \\
\hline & Mais de 30 & 8 & $13,3 \%$ \\
\hline \multicolumn{2}{|c|}{ Pontuação do teste de Fagerström } & 60 & $5(3 ; 6)$ \\
\hline
\end{tabular}

FONTE: O autor (2019). 
Na tabela contendo a comparação entre orientação presente ou ausente durante a hospitalização (Tabela 4), há diferença estatisticamente significativa entre os grupos para a variável "grau de motivação" ( $\mathrm{p}=0,03)$. Dos 21 pacientes que estavam no grau de motivação de ação, 19 receberam orientação, o que representa $44,2 \%$ de todos os pacientes que foram orientados. Entre os pacientes com orientação ausente, 76,5\% estavam nos graus mais baixos de motivação para cessação do tabagismo (pré-contemplação e contemplação).

Tabela 4 - Comparação entre orientação presente e ausente durante a hospitalização $(n=60)$

\begin{tabular}{l|l|c|c|c|c}
\hline \multicolumn{2}{l|}{ Variável } & Número de pacientes & $\begin{array}{c}\text { Orientação presente } \\
(\mathbf{n}=\mathbf{4 3})\end{array}$ & $\begin{array}{c}\text { Orientação ausente } \\
(\mathbf{n}=\mathbf{1 7})\end{array}$ & P-valor \\
\hline \multirow{5}{*}{ Grau de motivação } & Pré-contemplação & 13 & $7(16,3 \%)$ & $6(35,3 \%)$ & $7(41,2 \%)$ \\
& Contemplação & 19 & $12(27,9 \%)$ & $1(5,9 \%)$ & 0,03 \\
& Preparação & 6 & $5(11,6 \%)$ & $2(11,8 \%)$ & \\
& Ação & 21 & $19(44,2 \%)$ & $1(5,9 \%)$ & \\
& Manutenção & 1 & 0 & \\
\hline
\end{tabular}

FONTE: O autor (2019).

$\mathrm{Na}$ comparação entre orientação correta e incorreta durante a hospitalização (Tabela 5), houve diferença estatística $(p=0,026)$ entre os grupos em relação ao tipo de orientação recebida durante a hospitalização - motivacional ou motivacional com farmacoterapia. Sendo que, entre os pacientes orientados incorretamente, $18(81,8 \%)$ foram orientados somente de forma motivacional. Além disso, houve significância estatística $(\mathrm{p}=0,012)$ entre os pacientes que referiram dificuldade de não fumar em locais proibidos, dado que $18(81,8 \%)$ dos pacientes que foram orientados de forma incorreta, possuíam essa dificuldade.

Tabela 5 - Comparação entre orientação correta e incorreta durante a hospitalização $(n=60)$.

\begin{tabular}{|c|c|c|c|c|c|}
\hline \multicolumn{2}{|l|}{ Variável } & $\begin{array}{c}\text { Número de } \\
\text { pacientes }\end{array}$ & $\begin{array}{c}\text { Orientação correta } \\
(\mathbf{n}=21)\end{array}$ & $\begin{array}{c}\text { Orientação incorre- } \\
\text { ta }(\mathbf{n}=\mathbf{2 2})\end{array}$ & P-valor \\
\hline \multirow{5}{*}{ Grau de motivação } & Pré-contemplação & 7 & $3(14,3 \%)$ & $4(18,2 \%)$ & \multirow{5}{*}{0,79} \\
\hline & Contemplação & 12 & $5(23,8 \%)$ & $7(31,8 \%)$ & \\
\hline & Preparação & 5 & $2(9,5 \%)$ & $3(13,6 \%)$ & \\
\hline & Ação & 19 & $11(52,4 \%)$ & $8(36,4 \%)$ & \\
\hline & Manutenção & 0 & $0(0 \%)$ & $0(0 \%)$ & \\
\hline \multirow{2}{*}{$\begin{array}{l}\text { Tipo de orientação durante } \\
\text { a hospitalização }\end{array}$} & Motivacional & 28 & $10(47,6 \%)$ & $18(81,8 \%)$ & \multirow[b]{2}{*}{0,026} \\
\hline & $\begin{array}{l}\text { Motivacional + } \\
\text { farmacoterapia }\end{array}$ & 15 & $11(52,4 \%)$ & $4(18,2 \%)$ & \\
\hline \multicolumn{2}{|c|}{ Dificuldade de não fumar em locais proibidos } & 27 & $9(42,9 \%)$ & $18(81,8 \%)$ & 0,012 \\
\hline
\end{tabular}

FONTE: O autor (2019).

\section{DISCUSSÃO}

Conforme os resultados apresentados, observou-se que - dentre os pacientes tabagistas $-65 \%$ possuía escolaridade inferior a 8 anos, condizente com a literatura, a qual demonstra que a prevalência do tabagismo diminui com o aumento da escolaridade ${ }^{4-6}$.

Constata-se que $80 \%$ dos pacientes relataram ter recebido orientação no período pré-hospitalização e $73 \%$ possuía conhecimento sobre tratamento farmacológico e cessação de tabagismo. Na literatura, 73\% dos pacientes que procuraram tratamento, conseguiram obtê-lo, evidenciando que uma porcentagem de pacientes superior a $20 \%$ não possui assistência adequada afim de cessar o tabagismo ${ }^{5}$.

Classicamente é descrito que aproximadamente
20,7\% dos fumantes adquirem DPOC, o que se aproxima com os dados encontrados neste presente estudo, na qual $16,7 \%$ da amostra possuía DPOC ${ }^{18}$.

A U.S. Preventive Services Task Force (USPSTF) sugere - com grau de recomendação A (alto grau de certeza de que o benefício líquido é substancial) - que os médicos perguntem a todos os adultos sobre o tabagismo, que os aconselhem sobre o uso do tabaco e que forneçam intervenções motivacionais e farmacoterápicas ${ }^{19}$. Confrontando essa recomendação com nossos achados, observa-se uma ocorrência de orientação de apenas 71,7\%, o que demonstra uma quantidade insuficiente de orientação afim de cessar o tabagismo, dado que os pacientes hospitalizados estão mais suscetíveis às orientações passadas pelos médicos e agentes de saúde .

Comparando os grupos de orientados e não orienta- 
dos com relação ao grau de motivação para cessação do tabagismo, observou-se que o grupo com maior porcentagem dentre os orientados foram os que estão em fase de ação $(44,2 \%)$. Isso indica que os pacientes que estabeleceram um risco entre o tabagismo e a doença que causou sua hospitalização, ou entre a constatação de que o tabagismo seja prejudicial à sua saúde, já iniciaram um planejamento de cessar o tabagismo. O médico, notando isso, orienta com maior frequência a cessação utilizando a intervenção motivacional ou motivacional juntamente à farmacoterapia.

No entanto, muitas vezes a orientação realizada é feita de maneira insuficiente, o que é evidenciado pela porcentagem de que $81,8 \%$ das orientações incorretas são do tipo motivacionais. Entende-se por esse fato que o médico muitas vezes tende a fazer apenas a orientação motivacional, deixando de realizar a abordagem farmacológica, a qual tem alta evidência científica de auxílio ao paciente quanto a cessação ao tabagismo ${ }^{19}$. O que reforça ainda mais esse achado é que os pacientes com dificuldade de não fumar em locais proibidos, ou seja, pacientes que possuem um nível importante de dependência, são os que menos receberam orientação corretamente $(81,8 \%)$.

Com relação aos pacientes não orientados durante a hospitalização, $76,5 \%$ deles estavam nos graus mais baixos de motivação (pré-contemplação e contemplação), o que pode aventar a ideia de que os pacientes que não possuíam o insight de que cessar o tabagismo é importante para sua saúde não são orientados pelos médicos. Porém, esse conceito precisa ser revisto pelos profissionais, dado que a literatura atual afirma que a orientação baseada em estágios de motivação não é superiora em comparação a orientações não baseadas nesses estágios ${ }^{20}$.

O presente estudo apresentou como limitações o tamanho da amostra, a análise em um único centro médico hospitalar, assim como a inclusão de pacientes admitidos exclusivamente em um hospital terciário e a não abrangência de pacientes de todos os níveis socioeconômicos.

\section{CONCLUSÃO}

Conclui-se com essa análise que o grau de dependência de nicotina dos pacientes tabagistas hospitalizados no CHC/UFPR é médio (cinco pontos no teste de Fagerström). Além disso, encontramos que a maior parte das orientações $(51,2 \%)$ são realizadas de forma incorreta.

Participação dos autores: Os acadêmicos do curso de medicina, Gabriel Bordignon e Roddie Moraes Neto, foram responsáveis pela coleta dos dados, análise dos dados e elaboração do texto do artigo. O orientador e autor principal, Gustavo Lenci Marques, foi responsável pela revisão do texto final e orientação do projeto.

\section{REFERÊNCIAS}

1. Silva L, Araújo A, Queiroz A, Sales M, Sbpt M. Controle do tabagismo: desafios e conquistas. J Bras Pneumol. 2016;42(4):290-8. doi:10.1590/s1806-37562016000000145.

2. Pinto MT, Pichon-Riviere A, Bardach A. Estimativa da carga do tabagismo no Brasil: Mortalidade, morbidade e custos. Cad Saúde Pública. 2015;31(6):1283-97. doi:10.1590/0102$311 X 00192013$.

3. Carter BD, Abnet CC, Feskanich D, et al. Smoking and mortality-beyond established causes. N Engl J Med. 2015;372(7):631-40. doi:10.1056/NEJMsa1407211.

4. Instituto Brasileiro de Geografia e Estatística (IBGE). Pesquisa Nacional de Saúde 2013. IBGE; 2014 [citado 20 ago. 2019]. Disponível em: http://biblioteca.ibge.gov.br/ visualizacao/livros/liv91110.pdf. Published 2014.

5. Malta DC, Oliveira TP, Vieira ML, Almeida L, Szwarcwald CL. Uso e exposição à fumaça do tabaco no Brasil: resultados da Pesquisa Nacional de Saúde 2013. Epidemiol Serv Saúde. 2015;24(2):239-48. doi:10.5123/S167949742015000200006.

6. Brasil. Ministério da Saúde. Vigitel Brasil 2018: vigilância de fatores de risco e proteção para doenças crônicas por inquérito telefônico. Brasília: Ministério da Saúde; 2019 [citado 15 ago. 2019]. Disponível em: https://portalarquivos2.saude.gov.br/ images/pdf/2019/julho/25/vigitel-brasil-2018.pdf.

7. Pinto M, Bardach A, Palacios A, et al. Carga do tabagismo no Brasil e benefício potencial do aumento de impostos sobre os cigarros para a economia e para a redução de mortes e adoecimento. Cad Saúde Pública. 2019;35(8):e00129118. doi:10.1590/0102-311X00129118.

8. Instituto Nacional do Câncer. Protocolo Clínico e Diretrizes Terapêuticas - Dependência à Nicotina. Rio de Janeiro: INCA; 2014 [citado 20 ago. 2019]. Disponível em: http://www.saude. sp.gov.br/resources/cve-centro-de-vigilancia-epidemiologica/ areas-de-vigilancia/doencas-cronicas-nao-transmissiveis/doc/ dent/2014_diretrizes_inca_terapia_nicotina.pdf. Published 2014.

9. Reichert J, Araújo AJ de, Gonçalves CMC, et al. Diretrizes para cessação do tabagismo - 2008. J Bras Pneumol. 2008;34(10):845-80. doi:10.1590/S180637132008001000014 .

10. European Network for Smoking and Tobacco Prevention aisbl (ENSP). Guidelines for treating tobacco dependence. Brussels: ENSP; 2018 [cited 2019 Aug 18]. Available from: http://elearning-ensp.eu/pluginfile.php/1052/mod_resource/ content/2/guidelines_2018_english.pdf.

11. Vogelmeier CF, Criner GJ, Martinez FJ, et al. Global strategy for the diagnosis, management, and prevention of chronic obstructive lung disease 2017 report. Am J Respir Crit Care Med. 2017;195(5):557-82. doi:10.1164/rccm.201701-0218PP.

12. Cahill K, Stevens S, Perera R, Lancaster T. Pharmacological interventions for smoking cessation: an overview and network meta-analysis. Cochrane Database Syst Rev. 2013;(5). doi:10.1002/14651858.CD009329.pub2. 
13. Associação Médica Brasileira. Agência Nacional de Saúde Suplementar. Diretrizes Clínicas na Saúde Suplementar Tabagismo. Brasília: AMB; 2011 [citado 19 ago. 2019].. Disponível em: https://diretrizes.amb.org.br/ans/tabagismo. pdf.

14. de Oliveira GMM, Mendes M, Dutra OP, et al. 2019: Recommendations for reducing tobacco consumption in Portuguese-speaking countries-positioning of the Federation of Portuguese Language Cardiology Societies. Arq Bras Cardiol. 2019;112(4):477-86. doi:10.5935/abc.20190071.

15. Liebmann EP, Scheuermann TS, Faseru B, Richter KP. Critical steps in the path to using cessation pharmacotherapy following hospital-initiated tobacco treatment. BMC Health Serv Res. 2019;19(1):246. doi:10.1186/s12913-019-4059-4.

16. Stefan MS, Pack Q, Shieh MS, et al. The association of nicotine replacement therapy with outcomes among smokers hospitalized for a major surgical procedure. Chest. 2020;157(5):1354-1361.doi:10.1016/j.chest.2019.10.054.
17. Rigotti NA, Clair C, Munafò MR, Stead LF. Interventions for smoking cessation in hospitalised patients. Cochrane Database Syst Rev. 2012;(5). doi:10.1002/14651858.CD001837.pub3.

18. Lange P, Vestbo J. The Copenhagen City Heart Study Experience and its Key Contributions to Chronic Obstructive Pulmonary Disease. Barcelona Respir Netw. 2018;4(4). doi:10.23866/brnrev:2017-0022.

19. Siu AL. Behavioral and Pharmacotherapy Interventions for Tobacco Smoking Cessation in Adults, Including Pregnant Women: U.S. Preventive Services Task Force Recommendation Statement. Ann Intern Med. 2015;163(8):622-34. doi:10.7326/ M15-2023.

20. Cahill K, Lancaster T, Green N. Stage-based interventions for smoking cessation. Cochrane Database Syst Rev. 2010;(11). doi:10.1002/14651858.CD004492.pub4.

Recebido:13/03/2020

Aceito: 17/04/2020 